Article

\title{
The Effect of GPRC5a on the Proliferation, Migration Ability, Chemotherapy Resistance, and Phosphorylation of GSK-3 $\beta$ in Pancreatic Cancer
}

\author{
Bin Liu ${ }^{(0)}$, Hai Yang, Christian Pilarsky * $\mathbb{C}^{\mathbb{D}}$ and Georg F. Weber \\ Department of Surgery, Universitätsklinikum Erlangen, Krankenhausstraße 12, 91054 Erlangen, Germany; \\ Liu.Bin@uk-erlangen.de (B.L.); Hai.Yang@uk-erlangen.de (H.Y.); Georg.Weber@uk-erlangen.de (G.F.W.) \\ * Correspondence: christian.pilarsky@uk-erlangen.de
}

Received: 29 April 2018; Accepted: 25 June 2018; Published: 26 June 2018

\begin{abstract}
Pancreatic cancer ( $\mathrm{PaCa}$ ) is the fourth leading cause of cancer-related death, and personalized targeted cancer therapy is becoming a promising treatment strategy for $\mathrm{PaCa}$. The central approach of targeted therapy is to find a targetable key and an effective targeting method. In this study, the importance of GPRC5a (the G-protein-coupled receptor family C, member 5, group A) was identified using data mining methods based on published datasets. After analysis of the basic expression of GPRC5a in normal pancreas tissue and various PaCa cell lines, gene editing of GPRC5a in the human PaCa cell line MIA PaCa-2 and the mouse PaCa cell line TB32047 was performed using CRISPR/Cas9 (Clustered Regularly Interspaced Short Palindromic Repeats/CRISPR-associated proteins 9) to investigate the influence of GPRC5a on the proliferation and migration of PaCa cells as well as its effects on chemotherapy drug resistance. The results showed that GPRC5a was upregulated in PaCa tissues and various PaCa cell lines. Knockout of GPRC5a reduced the proliferation and migration ability of $\mathrm{PaCa}$ cell lines and suppressed the chemotherapy drug resistance of gemcitabine, oxaliplatin, and fluorouracil in PaCa cells. The phosphorylation of GSK-3 $\beta$ (Glycogen synthase kinase-3 $\beta$ ) was found to be upregulated in the MIA PaCa-2 and TB32047 cells after GPRC5a knockout. In conclusion, GPRC5a was upregulated in PaCa leading to an enhanced drug resistance in PaCa cells. These results provide for the first time a theoretical basis for the development of an improved $\mathrm{PaCa}$ targeted therapy.
\end{abstract}

Keywords: pancreatic cancer; GPRC5a; CRISPR/Cas9 system; GSK-3 $\beta$

\section{Introduction}

Pancreatic cancer $(\mathrm{PaCa})$ is one of few cancer types showing no improvement over the course of the 5-year survival rate [1]. PaCa was diagnosed in approximately 53,070 patients, with an estimated 41,780 deaths, in the United States in 2016 [2]. In China, the incidence of PaCa was in an upward trend from 2000 to 2011, and $\mathrm{PaCa}$ is one of the top 10 most common cancers in both men and women [3]. In Europe, PaCa is currently one of the most lethal cancers, with a 5-year survival of approximately $7 \%$. It is expected to rise to second place behind lung cancer by 2030 [4]. Surgery is currently the only potentially curative treatment for $\mathrm{PaCa}$ [5]. However, surgical treatment is subjected to some limitations, such as rapid disease progression, late diagnosis at advanced, unresectable stages, and inadequate response to current drug regimens [6,7]. Personalized targeted cancer therapy is a promising treatment strategy for $\mathrm{PaCa}$. The basic concept of targeted therapy is to find a targetable key in cancer as well as an effective targeting method $[8,9]$. In this study, we combined data-mining methods and the CRISPR/Cas9 system to research the correlation between one gene-the G-protein-coupled receptor family $\mathrm{C}$, member 5 , group A (GPRC5a) -and PaCa. We want to investigate the gene's biology functions in $\mathrm{PaCa}$ and its potential targeted value in personalized therapy. 
GPRC5a, also named retinoic acid-inducible 3 (RAI3), is a family member of the G-protein-coupled receptors, which is the largest protein superfamily with more than 700 genes in the human genome [10]. GPRC5a was originally identified in 1998 in a human oral squamous carcinoma cell line. GPRC5a is overexpressed in a variety of cancers, including colon cancer, breast cancer, and gastric cancer [11]. A previous study from our group showed higher expression levels of GPRC5a in PaCa tissues than in normal tissues [12]. Therefore, we thought to investigate GPRC5a as a potential gene of targeted therapy.

In this study, data-mining methods were used as a powerful supplement to prove the importance of GPRC5a in PaCa and the expression of GPRC5a in various PaCa cell lines was analyzed. We analyzed the potential functions of GPRC5a in PaCa with CRISPR/Cas9 system knockout cells and its relevance to chemoresistance.

\section{Results}

\subsection{The Data-Mining Analysis for GPRC5a in PaCa}

We identified 1673, 1692, 1848, 2393, 414, 2488, and 1835 differentially expressed genes (DEGs) in PaCa compared to normal tissue in the GSE15471, GSE16515, GSE19281, GSE22780, GSE28735, GSE32676, and GSE41372 datasets. A total of 85 genes were considered DEGs in all datasets (Figure 1A-C) and GPRC5a is one of the 85 DEGs (Table 1). Overall survival analysis was conducted to detect the relationship between GPRC5a expression and PaCa outcome. High mRNA expression of GPRC $5 a$ was associated with worse overall survival in both datasets (log-ranks $p$-value $=0.002845$ and 0.006623, Figure 1C,D) confirming our previous results [12]. Furthermore, "The Human Protein Atlas" was used to measure the basic expression level of GPRC5a in different human organs. The basic mRNA expression level of GPRC5a in the normal tissue of the pancreas was lower than that in other organs using the HPA (Human Protein Atlas) dataset, the GTEx (Genotype-Tissue Expression) dataset, and the FANTOM5 (The Functional Annotation of the Mammalian Genome 5) dataset (Figure 1F,G, HPA and FANTOM5 showed the same results). Moreover, the basic expression levels of GPRC5a in pancreatic cancer tissues at different stages were analyzed with the TCGA (The Cancer Genome Atlas) dataset. The results showed that the expression level of GPRC5a increased with higher stages of PaCa (Figure 2A,B).

Table 1. The expression levels of GPRC5a in seven microarray datasets. Compared with normal pancreas tissues, the expression level of GPRC5a was upregulated in pancreatic cancer tissues ( $p$-value $<0.05$ and $\log \mathrm{FC}<-1$ ).

\begin{tabular}{cccc}
\hline Microarray & $p$-Value & Student's $\boldsymbol{t}$-Value & $\operatorname{logFC}$ \\
\hline GSE15471 & $1.12 \times 10^{-12}$ & 8.689697 & 2.5051499 \\
GSE16515 & $1.08 \times 10^{-10}$ & 8.048017 & 4.3196152 \\
GSE19281 & $5.56 \times 10^{-3}$ & 3.6910787 & 2.28094556 \\
GSE22780 & 0.0186679 & 2.584605 & 2.44569319 \\
GSE28735 & $2.89 \times 10^{-12}$ & 8.059183 & 1.1751238 \\
GSE32676 & $1.68 \times 10^{-3}$ & 3.45 & 2.23 \\
GSE41372 & $2.34 \times 10^{-6}$ & 7.93 & 3.35 \\
\hline
\end{tabular}


A

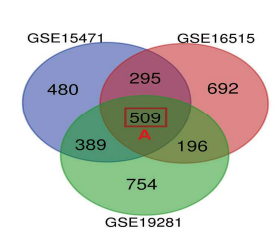

$\mathrm{F}$

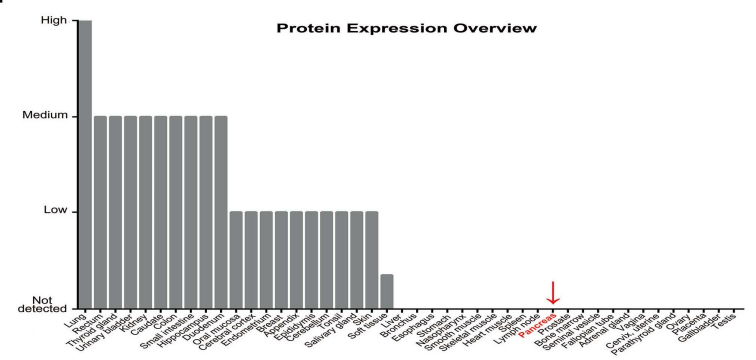

D

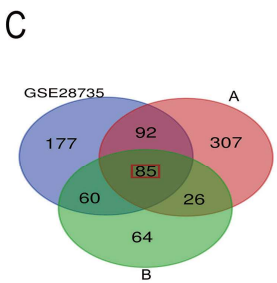

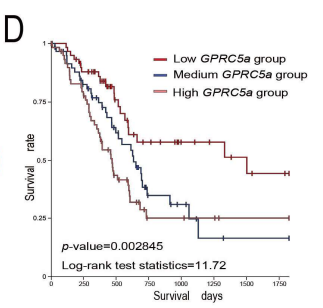

E

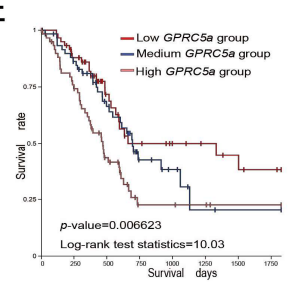

G

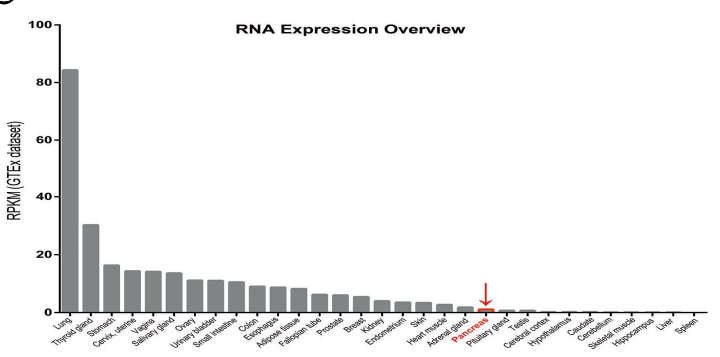

Figure 1. Data-mining analysis of GPRC5a in PaCa. (A-C) Identification of differentially expressed genes (DEGs) in seven mRNA expression-profiling datasets. GPRC5a is 1 of the 85 DEGs. The red frame named A means the DEGs among GSE15471, GSE16516 and GSE 19281. The red frame named B means the DEGs among GSE22780, GSE41372 and GSE 32676. The red frame in Figure 1C means the DEGs among GSE28735, red frame A and red frame B. (D,E) Prognostic value of GPRC5a in PaCa patients with different datasets. High mRNA-level expression of GPRC5a was associated with worse overall survival in the GDC (Genomic Data Commons) TCGA PaCa (223 samples) (D) and the TCGA PaCa (196 samples) datasets (E). (F,G) Basic expression levels of GPRC5a in different organs. The protein-level expression of GPRC5a was relatively low in the pancreas compared with that in the other 43 organs (F), similar to the mRNA expression of GPRC5a (G).

\subsection{Expression of GPRC5a in $\mathrm{PaCa}$ Cell Lines}

To further investigate the expression of GPRC5a in PaCa, we analyzed the protein expression levels in the normal pancreas HDPEE6E7 cell line and different PaCa cell lines by Western blot and immunofluorescence assay. The results showed that the protein expression level of GPRC5a in PaCa cell lines was significantly higher as compared to the normal pancreas cell line. Q-PCR analysis similarly showed a higher relative expression of GPRC5a in the PaCa cell lines as compared to the normal pancreas cell line (Figure 2C,D). Immunofluorescent analysis showed overexpression of GPRC5a in PaCa cell lines as compared to the normal pancreas HDPEE6E7 cell line (Figure 2E).

\subsection{Inhibition of the Proliferation and Migration Ability of TB32047 and MIA PaCa-2 Cells by Knockout of GPRC5a}

We next analyzed GPRC5a's function in the MIA PaCa-2 and KPC (Pdx1-Cre; K-Ras ${ }^{+/ L S L G 12 D ;}$ p53 $3^{\mathrm{R} 172 \mathrm{H} /+}$ ) mouse-model-derived TB32047 cell lines. The CRISPR/Cas9 gene editing system was used to knockout GPRC5a in those two cell lines. Western blot and sequencing were performed to detect the knockout effect. The results showed no protein expression in knockout MIA PaCa-2 and TB32047 cells (Figure 3A,C). In/del mutations in the sequence of GPRC5a were detected in all cells with GPRC5a knockout (Figure 3B,D). The proliferation ability of cells with GPRC5a knockout was compared with wild-type (WT) and negative control cells. The results showed that cells with GPRC5a knockout grew slower as compared to negative control and wild-type cells (Figure 3E,F). Furthermore, the migration ability of cells with GPRC5a knockout was detected. The results showed that the migration ability of cells with GPRC $5 a$ knockout was lower as compared to negative control and wild-type cells (Figure 3G,H). 
A

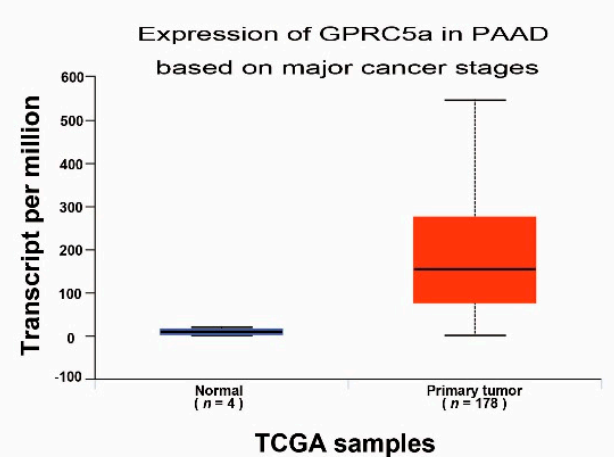

C

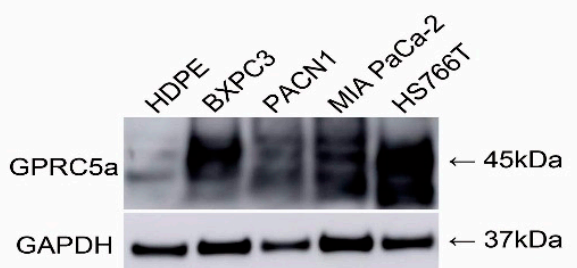

B

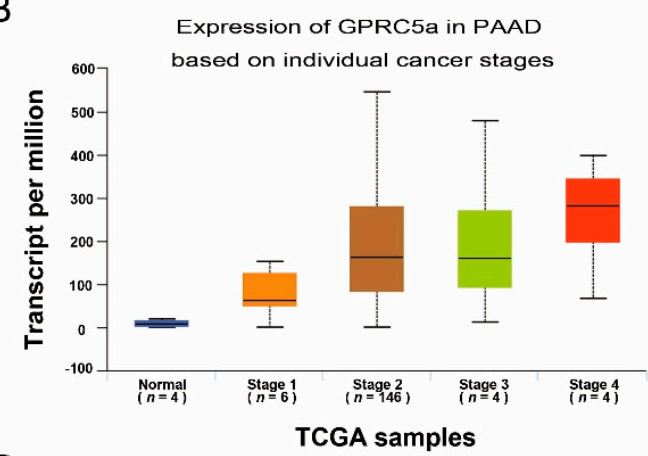

D

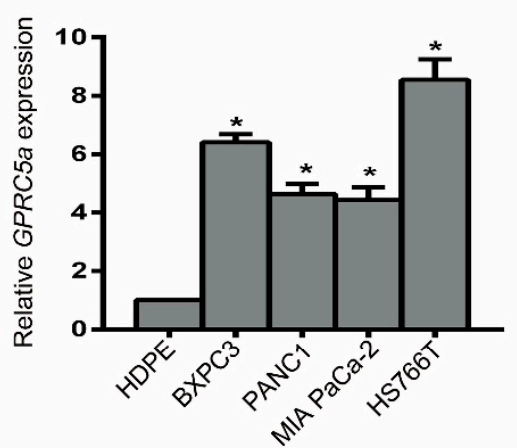

E

HDPE
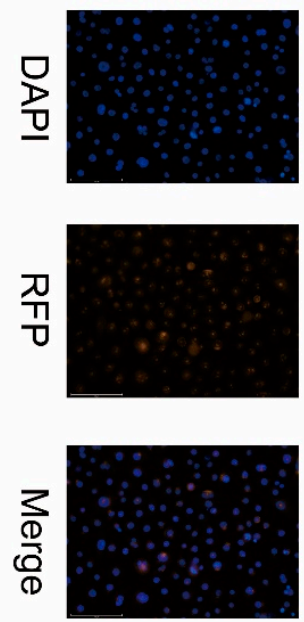

BXPC3
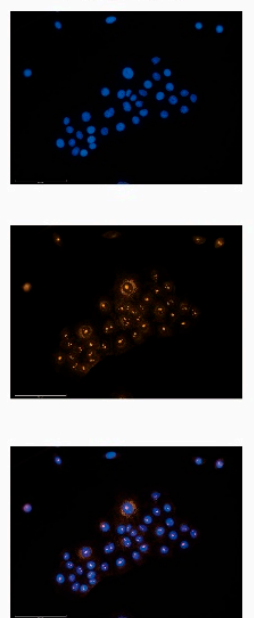

PANC1
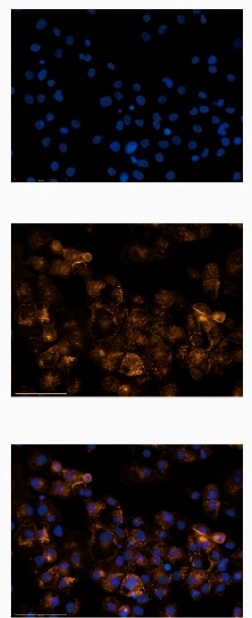

MIA PaCa-2
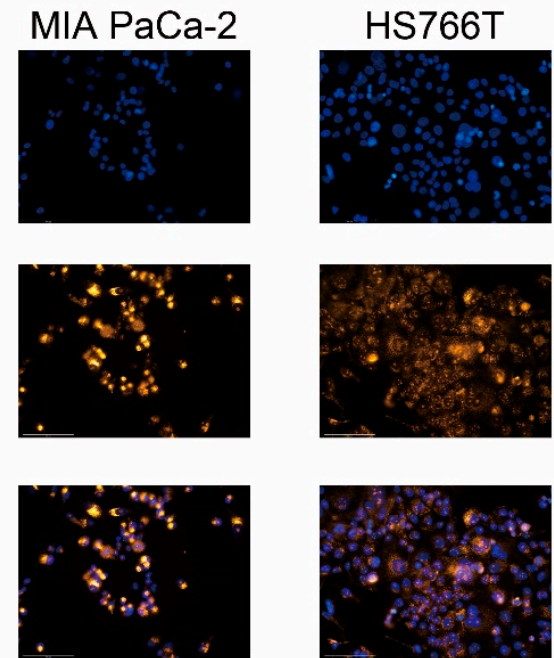

Figure 2. GPRC5a expression levels in normal pancreas and PaCa (PAAD) tissue and cell lines. (A,B) The data-mining analysis showed that GPRC5a was significantly upregulated in PaCa (PAAD) samples with an association of increased expression in higher malignant tumor stages $(p<0.05)$. $(\mathbf{C}, \mathbf{D})$ The Western blot and qPCR results showed that the basic protein-level expression of GPRC5a in $\mathrm{PaCa}$ cell lines was higher than that in the HPDEE6E7 pancreas cell line. (E) Immunofluorescence assay showed upregulated expression of GPRC5a in PaCa cell lines (200×). The error bars were from multiple samples $(n)$ measurement results $(\mathbf{A}, \mathbf{B})$ or three experimental measurements $(\mathbf{C}, \mathbf{D}) .{ }^{*} p$-value $<0.05$. 
A

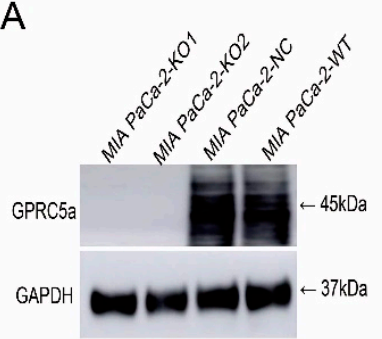

E

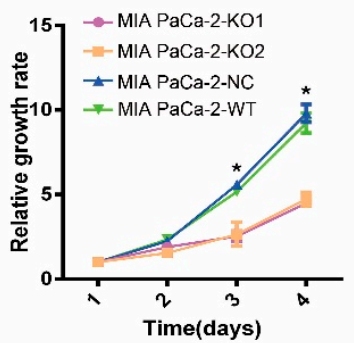

B

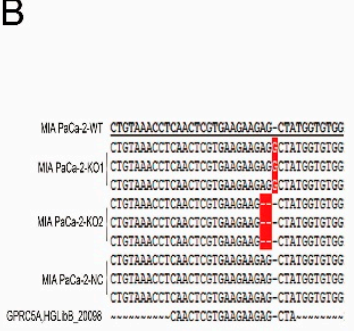

$\mathrm{F}$

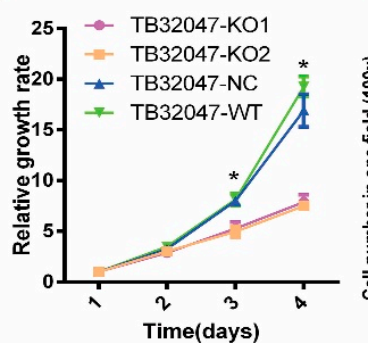

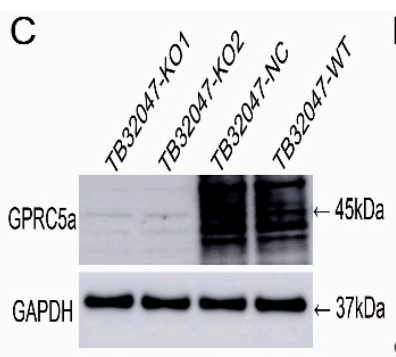

D

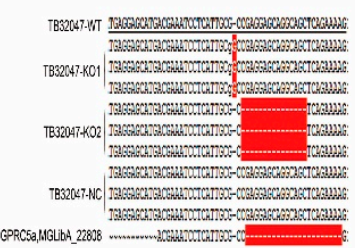

G

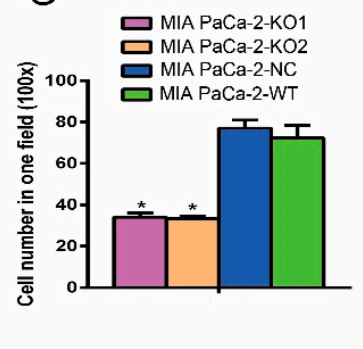

$\mathrm{H}$

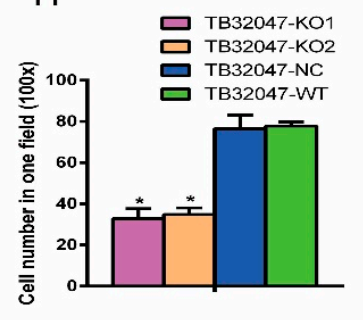

Figure 3. Influence of GPRC5a knockout on the proliferation and migration ability in MIA PaCa-2 and TB32047 cells. (A-D) Western blot and sequencing results showing the GPRC5a knocked out in MIA $\mathrm{PaCa}-2-\mathrm{KO} 1 / 2$ and TB32047-KO1/2 (Red highlight area means the different part in comparison of sequencing sequence). (E,F): The knockout of GPRC5a inhibited the proliferation ability of MIA PaCa-2 and TB32047 cells. $(\mathbf{G}, \mathbf{H})$ GPRC5a knockout inhibited the migration ability of MIA PaCa-2 (G) and TB32047 cells $(\mathbf{H}) .{ }^{*} p$-value $<0.05$.

\subsection{Inhibition of the Chemotherapy Drug Resistance in TB32047 and MIA PaCa-2 Cells by Knockout of GPRC5a}

We analyzed the response to the commonly used drugs 5-fluorouracil, gemcitabine, and oxaliplatin in the GPRC5a knockout system. In the GPRC5a knockout cell line, we observed a reduced resistance against these drugs compared to the WT cell line or the negative control cell line (Table 2, Figures 4 and S1).

Table 2. The EC50 values and 95\% CI (Confidence Interval) of chemotherapy drugs in cells.

\begin{tabular}{ccccccc}
\hline \multirow{2}{*}{ Cells } & \multicolumn{2}{c}{ Gemcitabine $(\mathbf{n M})$} & \multicolumn{2}{c}{ Oxaliplatin $(\boldsymbol{\mu M})$} & \multicolumn{2}{c}{ Fluorouracil $(\boldsymbol{\mu M})$} \\
\cline { 2 - 7 } & EC50 & $\mathbf{9 5 \%}$ CI & EC50 & $\mathbf{9 5 \% ~ C I ~}$ & EC50 & $\mathbf{9 5 \% ~ C I ~}$ \\
\hline MIA PaCa-2-KO1 & 0.455 & $0.305-0.605$ & 0.224 & $0.169-0.300$ & 4.642 & $3.577-6.340$ \\
MIA PaCa-2-KO2 & 0.476 & $0.322-0.665$ & 0.357 & $0.210-0.661$ & 4.502 & $3.720-5.525$ \\
MIA PaCa-2-NC & 1.458 & $1.128-1.938$ & 1.015 & $0.628-2.011$ & 6.675 & $5.406-8.424$ \\
MIA PaCa-2-WT & 1.546 & $1.122-2.146$ & 1.171 & $0.614-4.312$ & 6.777 & $5.321-9.061$ \\
TB32047-KO1 & 0.240 & $0.212-0.268$ & 1.661 & $1.378-2.000$ & 0.962 & $0.772-1.179$ \\
TB32047-KO2 & 0.274 & $0.242-0.306$ & 2.084 & $1.487-2.931$ & 0.828 & $0.666-1.024$ \\
TB32047-NC & 0.384 & $0.345-4.266$ & 5.774 & $5.309-6.281$ & 1.894 & $1.713-2.092$ \\
TB32047-WT & 0.413 & $0.353-0.486$ & 6.178 & $5.589-6.830$ & 1.947 & $1.743-2.168$ \\
\hline
\end{tabular}

The EC50 values of Gemcitabine and Oxaliplatin in cells with GPRC5a knockout accounted for only $30-40 \%$ of that in wild-type (WT) cells. The EC50 values of Fluorouracil in cells with GPRC5a knockout accounted for 50-75\% of that in negative control (NC) and WT cells. Values keep three decimal places. 


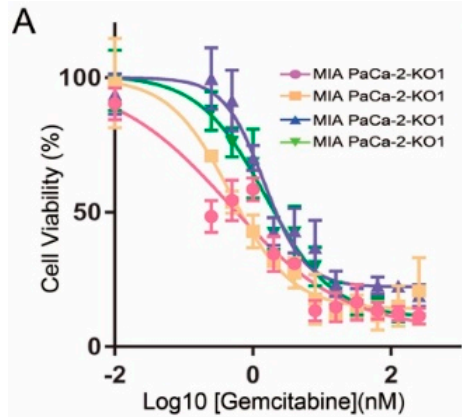

D

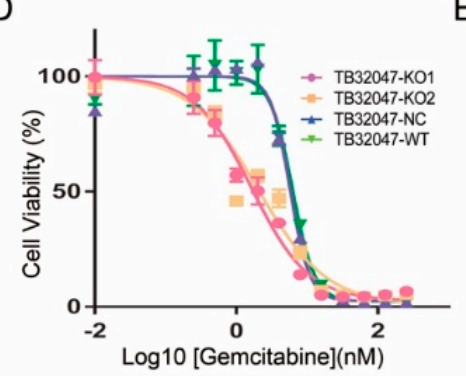

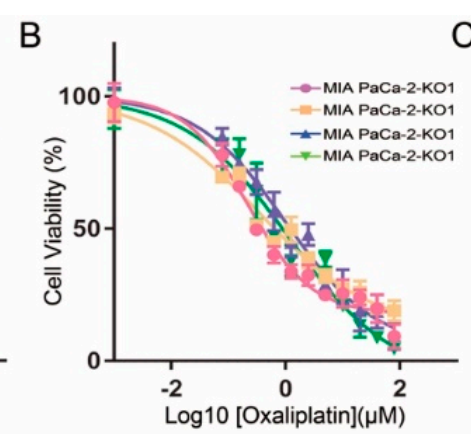

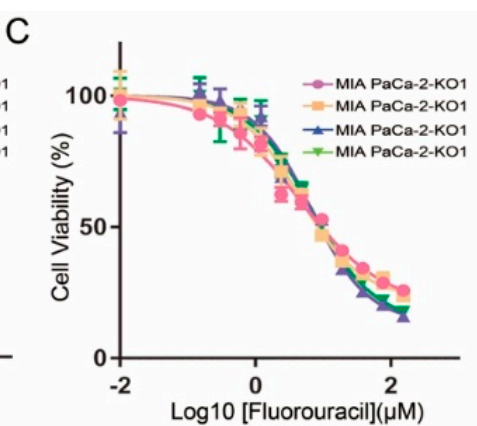

E

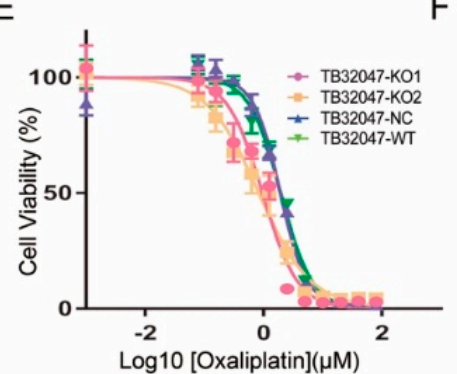

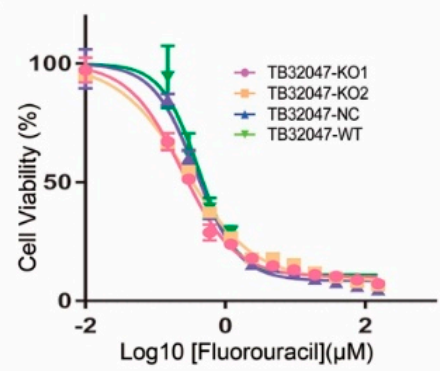

Figure 4. Knockout of GPRC5a suppressed chemotherapy drugs resistance in MIA PaCa-2 and TB32047 cells. (A-C) shows the EC50 (Concentration for 50\% of maximal effect) assay of gemcitabine, oxaliplatin, and fluorouracil in the MIA PcCa-2 cell line. (D-F) shows the same results in the TB32047 cell line.

\subsection{Knockout of GPRC5a Promotes the Phosphorylation of GSK-3ß at Ser9 in TB32047 and MIA PaCa-2 Cells}

The knockout of GPRC5a led to an upregulation of the phosphorylation of GSK-3 $\beta$ (Ser9) (Glycogen synthase kinase- $3 \beta$ )in MIA PaCa-2 and TB32047 cells. There was no significant change in the expression of GSK-3 $\beta$, GSK-3 $\alpha$, $\beta$-Catenin, PPAR $\gamma$ (Peroxisome Proliferator-Activated Receptor $\gamma$ ), and Erk1/2 (extracellular signal-regulated kinase 1/2) or in its activated phosphorylation at Thr202/Tyr204 (Figures 5 and S2).

A

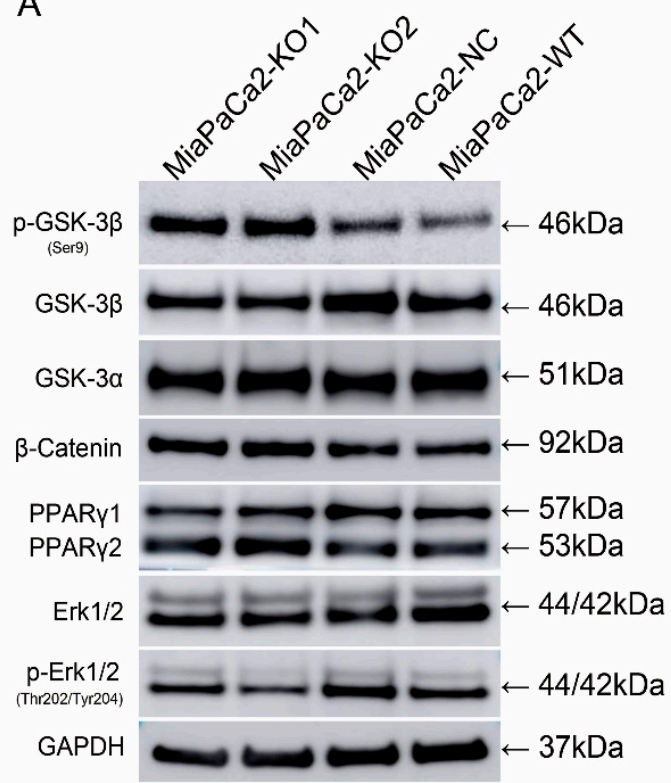

B

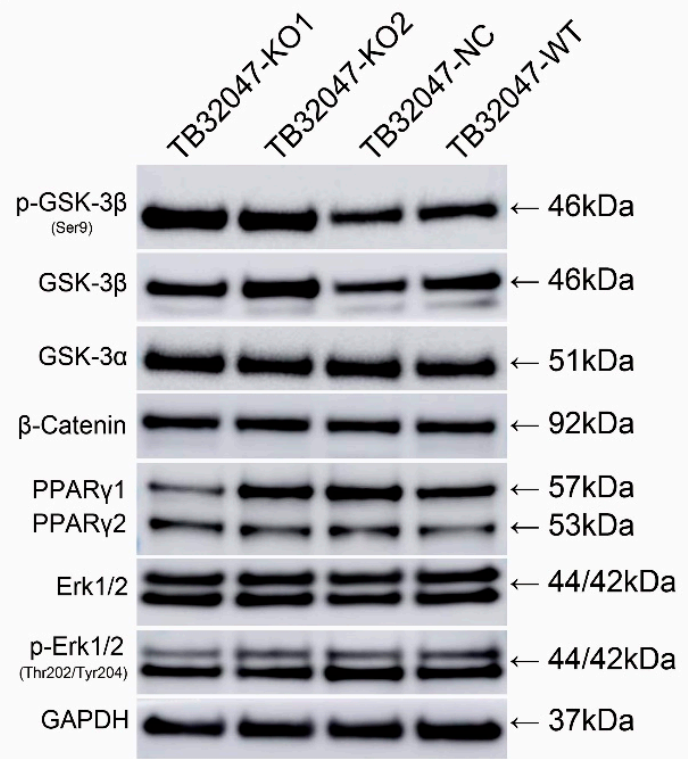

Figure 5. Knockout of GPRC5a promotes the phosphorylation of GSK-3 $\beta$ at Ser9. (A,B) shows GSK-3 $\beta$ and its related upstream and downstream protein expression with GPRC5a knockout in MIA PaCa-2 and TB32047, respectively. 


\section{Discussion}

G-protein-coupled receptors are the largest protein superfamily with more than 700 genes in the human genome [10] playing an important role in a variety of biological processes [13]. This protein superfamily also acts as a drug target in many different diseases and more than $40 \%$ of FDA (Food and Drug Administration)-approved drugs target GPCRs (G Protein-Coupled Receptors) or GPCR-associated pathways [14]. GPCRs also play an integral role in regulating and activating cancer-associated signaling pathways; therefore, they may be used as biomarkers for the early diagnosis of various cancers [15]. Further investigation of the pharmacological potential of GPCRs and their downstream regulators is required in order to develop therapies that can efficiently target cell signaling pathways in cancer $[16,17]$.

GPRC5a, also named RAI3 (Retinoic acid-induced protein 3), located on chromosome 12p13-p12.3, has been found to play significant roles in some biological processes, such as cell proliferation and the cell cycle. However, the influences of GPRC5a on different cancers vary. GPRC5a was reported to be strongly expressed in the lung $[18,19]$ and is regarded as a tumor suppressor in lung cancer as well as in head and neck squamous cell carcinoma [20]. However, there are many papers that have reported that the higher expression of GPRC5a correlated with a worse survival rate in colon cancer, breast cancer, and gastric cancer [11]. In our previous study, GPRC5a was reported to have lower expression levels in normal pancreas tissues and have a key influence on the proliferation of PaCa cells via STAT3 (Signal transducer and activator of transcription 3)-modulated pathways [12].

Data mining is a process of discovering patterns in large data sets and involves methods at the intersection of machine learning, statistics, and databases. It has been used in many biological applications [21,22]. The CRISPR/Cas9 gene editing system is a new technology to knock out genes at the DNA level [23]. As compared to RNAi, the pre-transcriptional knockout improves the functional analysis of a gene expression loss. In this study, data mining and CRISPR/Cas9 gene editing were used to investigate the role of GPRC5a from PaCa cell lines MIA PaCa-2 and TB32047.

Our data-mining approach confirmed previous results that GPRC5a is overexpressed in $\mathrm{PaCa}$ tissue and that this is associated with a shorter overall survival time [12].

It indicates that lowering the expression of GPRC5a may improve the prognosis and suggests that GPRC5a is an important gene in PaCa development. We then performed GPRC5a knockout experiments to elucidate the role of GPRC5a in PaCa.

Therefore, we analyzed the basic expression of GPRC5a in the normal pancreas cell line HPDEE6E7 and PaCa cell lines. The result showed that GPRC5a was upregulated in all PaCa cell lines at both the protein and mRNA levels. In order to analyze the function of GPRC5a in PaCa, GPRC5a was knocked out from the MIA PaCa-2 and TB32047 cell lines with the CRISPR/Cas9 gene editing system, the knockout effect was evaluated by Western blot and sequencing, and the influence of GPRC5a on proliferation and migration ability was analyzed. The results showed that cells with GPRC5a knockout grew or migrated slower than negative control and wild-type cells, indicating that the knockout of GPRC5a inhibited proliferation and migration ability in PaCa cell lines.

Secondly, we detected the drug resistance of chemotherapy after knockout of GPRC5a from the MIA PaCa-2 and TB32047 cell lines by EC50 assay. The results showed that knocking out GPRC5a from $\mathrm{PaCa}$ can suppress its resistance of the chemotherapy drugs gemcitabine, oxaliplatin, and fluorouracil. Gemcitabine is a synthetic pyrimidine nucleoside prodrug, which has been used in various carcinomas, including PaCa [24]. Oxaliplatin has also been used as chemotherapy drug in a variety of carcinomas, and it is worth noting that oxaliplatin is always used in combination with Fluorouracil [25]. Their inhibitory effect on PaCa has been proven in both experimental research and clinical treatment. However, all of these drugs have been reported to have side effects, which greatly limit their therapeutic applications. In this study, the drug sensitivity for gemcitabine, oxaliplatin, and fluorouracil by the knockout of GPRC5a could be increased, meaning that similar chemotherapy effects with lower concentrations of chemotherapy could be obtained in PaCa cells. As a consequence, the side effects may be significantly reduced if a GPRC5a knockout from PaCa patients' cancer cells is performed. 
This may be a potential targeted therapy, especially for PaCa patients with chemotherapy resistance. Interestingly, the inhibitory effects of the GPRC5a knockout were different in the three investigated drugs, whereby the sensitivity effect for Fluorouracil was smaller than that for the other two drugs.

It has been reported that the Gemcitabine resistance in $\mathrm{PaCa}$ has a close relationship with Akt-GSK3 $\beta$-Snail pathway activity, and it has been shown that oxaliplatin induces an epithelial-mesenchymal transition in cancers. Moreover, two main pathways, including GSK3 $\beta$ and PPAR $\gamma$ as hub genes, for the anti-cancer function of Fluorouracil in PaCa have been previously described [26,27]. In this study, we found that the activity of GSK3 $\beta$ was downregulated in cells with GPRC5a knockout, but no significant change in the expression of PPAR $\gamma$ could be observed. The PPAR $\gamma$-related pathways may have compensatory effects for the GPRC5a-suppressed Fluorouracil resistance. Of course, further in-depth studies are still needed on this issue.

GSK3 is a family of serine/threonine protein kinases comprising two highly conserved isoforms, GSK $3 \alpha$ and GSK3 $\beta$, which show approximately $85 \%$ overall homology and $98 \%$ homology in their kinase domains, respectively [28]. Of the two GSK3 isoforms, GSK3 $\beta$ has been studied most as the functional redundancy of the two isoforms in regulating the canonical Wnt/ $\beta$-Catenin pathway $[26,29]$. In $\mathrm{PaCa}$, it has been reported that inhibiting GSK3 $\beta$ activity with inhibitors or RNAi can preferentially attenuate the survival and proliferation of tumor cells and induce them to undergo apoptosis [30,31]. GSK3 $\beta$ also has a close relationship with therapy resistance [26]. Others showed that a GSK3 $\beta$-specific pharmacological inhibitor, named AR-A014418, given in combination with chemotherapeutic agents, had additive and synergistic effects [32]. Many studies show that GSK3 $\beta$ participates in chemotherapy, radiotherapy, and targeted therapies via multiple molecular pathways [33]. In this study, we found that p-GSK-3 $\beta$ at Ser9, the inactivated state of GSK3 $\beta$, was upregulated in cells with GPRC5a knockout. However, the total expression amount of GSK3 $\beta$ and GSK3 $\alpha$ remained unchanged. This shows that the phosphorylation state of GSK-3 $\beta$ has an inner relationship with GPRC $5 a$ expression. In addition, we detected the upstream- and downstream-related proteins in the main GSK-3 $\beta$ pathway. $\beta$-Catenin is the main downstream gene in the Wnt/GSK-3 $\beta / \beta$-Catenin pathway. The unchanged expression of $\beta$-Catenin indicates that GPRC5a has little influence on this pathway. Erk $1 / 2$ is the upstream gene of the Erk/GSK-3 $\beta$ pathway. In our previous study, Erk1/2 and p-Erk1/2 were not regulated with GPRC5a by RNAi interference. In this study, we confirmed these results that GPRC5a cannot regulate Erk1/2 or its phosphorylation state. The results suggested that GPRC $5 a$ may affect GSK-3 $\beta$ activity by promoting the GSK-3 $\beta$ inactivity phosphorylation state at Ser9. However, further investigations need to be conducted in the future to understand the role of GPRC $5 a$ in the regulation of GSK-3 $\beta$ activity.

\section{Methods}

\subsection{Data-Mining Analysis}

An Expression Omnibus database (GEO) dataset, in which a comparison between normal and PaCa tissue was performed, was identified (GSE16515 [34], GSE15471 [35], GSE28735 [36], GSE19281 [37], GSE22780 [38], GSE32676 [39], and GSE41372 [40]).

The seven gene expression datasets include 166 cancer tissue samples and 127 normal tissue samples from PaCa patients. All these datasets were generated on Affymetrix chips and uploaded by independent groups.

GEO2R is an online tool (https:/ / www.ncbi.nlm.nih.gov/geo/geo2r) which can compare different groups in a GEO series. In this study, GEO2R was used to identify differentially expressed genes (DEGs) between PaCa and normal tissue samples. The PaCa samples were regarded as the "cancer group" while the normal samples were regarded as the "control group". The genes for which the adjusted (Benjamini and Hochberg or False discovery rate) $p$-value was smaller than 0.01 and the absolute log fold-change was greater than 1 were selected.

The online tool "Calculate and draw custom Venn diagrams" (http:/ / bioinformatics.psb.ugent. be/webtools/Venn/) was employed to identify genes that were up or downregulated in all seven GEO 
datasets. As this tool is not able to deal with more than six groups in one time, the first three groups of gene expression datasets were selected, and then the remaining three groups of datasets were selected. Then, the other one group and the two above collection groups of datasets were analyzed together. After that, all DEGs were analyzed, and the expression level of GPRC5a in those seven gene expression microarrays was detected.

The UCSC Xena (https:/ / xenabrowser.net/) was adopted to perform overall survival analysis in PaCa. In this study, the TCGA Pancreatic Cancer (PAAD) and GDC TCGA Pancreatic Cancer datasets were selected to create Kaplan-Meier plots based on the gene expression level of GPRC5a. Hazard ratios with $95 \%$ confidence intervals and Log-rank $p$-values were recorded. The top one-third, middle one-third, and bottom one-third percentiles of GPRC5a expression values were considered to be the high, middle, and low groups, respectively. Then, the relationship between GPRC5a expression and survival time was detected.

Furthermore, the basic expression level of GPRC5a in different human organs was measured by "The Human Protein Atlas v17" [41]. There were three datasets in the website, including the HPA dataset (RNA-seq tissue data is reported as mean transcripts per million, corresponding to the mean values of the different individual samples from each tissue), the GTEx dataset (RNA-seq data is reported as median reads per kilobase per million mapped reads, generated by the Genotype-Tissue Expression project), and the FANTOM5 dataset (tissue data obtained by Cap Analysis of Gene Expression is reported as Tags Per Million, generated by the FANTOM5 project). The three datasets were combined and used in this study. In addition, the different expressions of GPRC5a between normal pancreas and PaCa tissues were measured. UALCAN is an easy online tool (http:/ / ualcan.path.uab.edu) for in-depth analyses of TCGA gene expression data [42]. It uses TCGA level 3 RNA-seq and clinical data from 31 cancer types. In this study, UALCAN was used to identify the up or downregulation of GPRC5a in PaCa.

\subsection{Cell Culture}

In this study, the human PaCa lines BxPC3, PANC1, HS766T, and MIA PaCa-2 and the normal pancreas cell line HDPEE6E7 were used. BXPC3, PANC1, HS766T, and MIA PaCa-2 were purchased from ATCC (American Type Culture Collection, Manassas, VA, USA). HDPEE6E7 was a gift from Ming-Sound Tsao, UHN (University Health Network), Toronto, ON, Canada. Primary mouse PaCa cell line TB32047 was provided by David Tuveson, CSHL (Cold Spring Harbor Laboratory, Cold Spring Harbor, NY, USA). All cell lines were grown in monolayer culture in a humidified atmosphere containing $5 \% \mathrm{CO}_{2}$ at $37^{\circ} \mathrm{C}$. The culture medium for BXPC 3 and PANC1 consisted of RPMI (Roswell Park Memorial Institute) Medium 1640 (Gibco, 21875-034, Waltham, MA, USA) with 10\% fetal bovine serum (FBS) (Gibco, A31608-01). HS766T and TB32047 cells were cultured in DMEM (Dulbecco's Modified Eagle's medium) medium (Gibco, 30966-021) with 10\% FBS. MIA PaCa-2 cells were cultured in DMEM medium with 10\% FBS and 2\% horse serum (Gibco, 16050-130). HDPEE6E7 cells were cultured in Keratinocyte-SFM (Keratinocyte-serum-free medium) medium (Gibco, 10724-011). All cells were harvested by $0.25 \%$ Trypsin-EDTA (Ethylenediaminetetraacetic acid) (Gibco, 25200-072).

\subsection{Western Blot}

Cells were lysed with RIPA-buffer (Radioimmunoprecipitation assay buffer). Gel electrophoresis was performed under reducing conditions with acrylamide gel and proteins were transferred to a nitrocellulose membrane. As primary antibodies, GPRC5a (NOVUS, NBP1-89743, Littleton, CO, USA), phosphorylation -GSK-3 $\beta$ (Cell Signaling, \#5558, Danvers, MA, USA ), GSK-3 $\beta$ (Cell Signaling, \#12456), GSK-3 $\alpha$ (Cell Signaling, \#4337), $\beta$-Catenin (Cell Signaling, \#8480), PPAR $\gamma$ (Cell Signaling, \#2443), Erk1/2 (Cell Signaling, \#4695), and phosphorylation Erk1/2 (Cell Signaling, \#4370) were used for detection. GAPDH (Cell Signaling Technology, 5175S) served as loading control. HRP (Horseradish peroxidase) -linked anti-rabbit IgG (Immunoglobulin G) (Cell Signaling Technology, 7074) was used as the secondary antibody. Quantification of signals was performed by an Amersham 
Imager 600 (Pittsburgh, PA, USA) with SignalFire Elite ECL Reagent (Cell Signaling Technology, 12757S). All Western blot assays were carried out individually two times, which validates the reliability of the results.

\subsection{Quantitative RT-PCR}

The isolation of RNA was performed with a NucleoSpin RNA Plus kit (Macherey-Nagel, \#740984.250, Düren, Nordrhein-Westfalen, Germany) and the cDNA was synthesized with a High Capacity cDNA Reverse Transcription Kit (Applied Biosystems, 00364942, Foster City, CA, USA). Quantitative RT-PCR was conducted with PowerUP SYBR Green Master Mix (Applied Biosystems, A25741) and analyzed by the $2^{-\triangle \Delta C t}$ method. GAPDH was used as a reference value. Primer sequences for amplification were GPRC5a-forward ( $5^{\prime}$-GCTGCTCACAAAGCAACGAA-3'), GPRC5a-reverse (5'-ATAGAGCGTGTCCCCTGTCT-3'), GAPDH-forward (5'-CTTTGGTATCGTGGAAGGACTC-3'), and GAPDH-reverse (5'-AGTAGAGGCAGGGATGATGT-3'). All primers were synthesized by Eurofins Genomics (Ebersberg, Germany).

\subsection{The CRISPR/Cas9 Gene Editing Knockout System}

In this study, GPRC5a was knocked out by the CRISPR/Cas9 gene editing system in MIA PaCa-2 and TB32047. pSpCas9(BB)-2A-GFP (PX458, addgene Plasmid \#48138) was used as a plasmid vector [43]. GPRC5a-human-sgRNA-forward (5'-caccCAACTCGTGAAGAAGAGCTA-3'), GPRC5a-human-sgRNA-reverse (5'-aaacTAGCTCTTCTTCACGAGTTG-3'), GPRC5a-mouse-sgRNAforward ( $5^{\prime}$-caccACGAAATCCTCATTGCGCCG-3'), GPRC5a-mouse-sgRNA-reverse ( $5^{\prime}$-aaacCGG CGCAATGAGGATTTCGT-3'), NC-human-sgRNA-forward (5'-caccATCGTATCATCAGCTAGCGC-3'), NC-human-sgRNA-reverse (5'-aaacGCGCTAGCTGATGATACGAT-3'), NC-mouse-sgRNA-forward (5'-caccGACCGGCCAACGGTAGCGGC-3'), and NC-mouse-sgRNA-reverse (5'-aaacGCCGCTACC GTTGGCCGGTC- $3^{\prime}$ ) were synthesized by Eurofins. The sgRNAs were designed based on the human and mouse CRISPR Knockout Pooled Library (GeCKO v2, Genome-scale CRISPR Knock-Out Version 2, http://genome-engineering.org/) [44]. Plasmid construction was done according to protocol and confirmed by sequencing. MIA PaCa-2 and TB32047 cells were transfected with GPRC5a knockout plasmid by Lipofectamine 3000 Transfection Reagent (Invitrogen, \#L3000015, Waltham, MA, USA) for $72 \mathrm{~h}$. Then, all the GFP (Green fluorescent protein)-positive cells were sorted by a fluorescence-activated cell sorter. Sixty GFP-positive cells were plated in one 96-well plate for selecting single clones. After a few days of culture, Western blot and sequencing were performed to detect the knockout effect. Sequencing was done by Eurofins, and all selected clones were sequenced at least five times. In this study, we set two of the positive single clones as "KO1" and "KO2" for each cell line. The cell transfected with NC-sgRNA was used as one of the control groups, which was named "NC". The other control group used wild-type cells and was named "WT".

\subsection{Proliferation Assay}

We used the cell-counting method to detect the proliferation ability of GPRC5a knockout cells and control cells. This method is achieved by fluorescence microscopy counting the number of cells in each well. Compared with a sampling count, it can count every cell in one well to avoid sampling bias. Cells were plated as $3 \times 10^{3}$ cells per well in grown medium on 96-well black plates (Corning, \#3603, Kaiserslautern, Germany) for four days. After 24,48 , and $72 \mathrm{~h}$ of proliferation, cells were stained with DAPI by NucBlue Live Cell Stain ReadyProbes reagent (Invitrogen, R37605) and imaged in 38 fields for each well by an Evos FL Auto 2 imaging system (Invitrogen, AMAFD2000). Images were counted by HCS studio cell analysis software V2.0 (Thermo, SX000041A, Waltham, MA, USA). All experiments were performed in duplicate and repeated at least three times. 


\subsection{Migration Assay}

A FluoroBlok ${ }^{\mathrm{TM}}$ Insert system (Corning, REF351152, Kaiserslautern Germany) was used to analyze the migration ability of GPRC5a knockout clones and the negative control clone. After being cultured for $24 \mathrm{~h}$ with free FCS medium, cells were plated as 25,000 cells per well in a FluoroBlok ${ }^{\mathrm{TM}}$ Insert, and the FluoroBlok ${ }^{\mathrm{TM}}$ Insert was put into a Multiwell 24 Well (Corning, REF353504). The medium in the up-well was free FCS medium and that in the down-well was grow medium with an extra $10 \%$ FCS. After $16 \mathrm{~h}$ of migration in an incubator, NucSpot Live 488 Nuclear Stain (Biotium, Cat40081, Fremont, CA, USA) was used. The cells, attached on the center of the membrane, were imaged in a random four fields for each well by the Evos FL Auto 2 imaging system (Invitrogen, AMAFD2000). The positive points in all images were counted manually.

\subsection{Chemotherapy Drugs Resistance Assay}

The influence of GPRC5a on PaCa was detected by the EC50 method. Cells were plated as $3 \times 10^{3}$ cells per well in grown medium on a 96-well black plate for $24 \mathrm{~h}$. Then, the chemotherapy drug was added to the well to final concentration. After cells were cultured for another $72 \mathrm{~h}$, they were counted with the same method used in the above proliferation assay. EC50 was calculated and plotted by GraphPad Version 7 (GraphPad Software, La Jolla, CA, USA). Gemcitabine, oxaliplatin, and fluorouracil were used for the EC50 assay in this study. These drugs were purchased from the pharmacy of the Universitätsklinikum Erlangen in ready-made solutions (Gemcitabine, $40 \mathrm{mg} / \mathrm{mL}$, Hexal AG, Holzkirchen, Freistaat Bayern, Germany), (oxaliplatin, $50 \mathrm{mg} / \mathrm{mL}$, Medac GmbH, Wedel, Niedersachsen, Germany), and (Fluorouracil, $5 \mathrm{mg} / \mathrm{mL}$, Accord Medical Ltd., London, UK). Except for the initial concentration, there were 11 gradient concentrations for each drug. The initial concentration was set to $0.01 \mathrm{nM}$ (gemcitabine), $0.01 \mu \mathrm{M}$ (oxaliplatin), and $0.01 \mu \mathrm{M}$ (fluorouracil). The gradient concentration range was $(0.25-256 \mathrm{nM})$ for gemcitabine, $(0.02-20 \mu \mathrm{M})$ for oxaliplatin, and $(0.15-153.6 \mu \mathrm{M})$ for fluorouracil. The concentration was doubly increased.

\subsection{Immunofluorescence Assay}

Cells were plated in 48-well plates and cultured to an appropriate density. After trypsinization, the cells were covered with $100 \mu \mathrm{L}$ of $4 \%$ warm formaldehyde. The cells were cultured for $15 \mathrm{~min}$ at room temperature. Wells were washed with PBS three times. Firstly, block buffer was added, and the cells were cultured for $60 \mathrm{~min}$ at room temperature. Then, $100 \mu \mathrm{L}$ of antibody buffer with $0.5 \%$ primary antibody GPRC5a (NOVUS, NBP1-89743) were added and the cells were cultured at $4{ }^{\circ} \mathrm{C}$ overnight. On the second day, the wells were washed with PBS three times before adding $100 \mu \mathrm{L}$ of antibody buffer with $0.5 \%$ secondary antibody Anti-rabbit IgG (Cell Signaling Technology, 4413S). After that, the wells were placed away from light and cultured for $2 \mathrm{~h}$ at room temperature. Then, the secondary antibody was washed away with PBS three times. Finally, the fluorescence assay was carried out by the Evos FL Auto 2 imaging system.

\subsection{Statistical Analysis}

Statistical analysis was performed with GraphPad Version 7 and Microsoft Excel Version 2017 (Microsoft, Seattle, WA, USA). A $t$-test was used in cell culture experiments. $p$-values $<0.05$ were considered statistically significant. The EC assay was calculated by GraphPad. After transforming concentrations to logarithms ( $\log 10)$, a $\log$ (inhibitor) versus response variable slope (four parameters) was used to calculate the EC50 and 95\% CI. All assays were carried out individually two times at least $(n \geq 2)$. 


\section{Conclusions}

In conclusion, GPRC5a is overexpressed in PaCa tissue and PaCa cell lines. The upregulation of GPRC5a in PaCa may be associated with a worse prognosis. Knockout of GPRC5a by the CRISPR/Cas 9 system can suppress proliferation and migration ability and may promote chemotherapy drug sensitivity in PaCa cells. GSK-3 $\beta$ activity was inhibited by upregulation of phosphorylation GSK-3 $\beta$ at Ser9 in cells with GPRC5a knockout. This study provides a reference for developing a targeted therapy for PaCa.

Supplementary Materials: The following are available online at http:/ /www.mdpi.com/1422-0067/19/7/1870/s1. Author Contributions: Conceptualization: B.L., C.P., and G.F.W. Data curation: B.L. Formal analysis: B.L. Funding acquisition: B.L. and C.P. Supervision: C.P. and G.F.W. Writing, original draft: B.L. Writing, review \& editing: B.L., H.Y., C.P., and G.F.W. All authors participated in the process of revision and finalizing the manuscript. All authors read and approved the final manuscript.

Funding: B.L. is funded by a China Scholarship Council (CSC) scholarship (No. 201406210072). All data mining source data are available on the appropriate Web sites.

Acknowledgments: We would like to thank all of the scientists who were able to put their software and data online. Without the efforts of the research community, the data mining part of this study would have been impossible. The present work was performed in fulfillment of the requirements for obtaining the degree "Dr. rer. biol. hum.".

Conflicts of Interest: The authors declare no conflict of interest.

\section{References}

1. New, M.; van Acker, T.; Long, J.S.; Sakamaki, J.I.; Ryan, K.M.; Tooze, S.A. Molecular Pathways Controlling Autophagy in Pancreatic Cancer. Front. Oncol. 2017, 7, 28. [CrossRef] [PubMed]

2. Siegel, R.L.; Miller, K.D.; Jemal, A. Cancer statistics, 2016. CA Cancer J. Clin. 2016, 66, 7-30. [CrossRef] [PubMed]

3. Chen, W.; Zheng, R.; Baade, P.D.; Zhang, S.; Zeng, H.; Bray, F.; Jemal, A.; Yu, X.Q.; He, J. Cancer statistics in China, 2015. CA Cancer J. Clin. 2016, 66, 115-132. [CrossRef] [PubMed]

4. De Angelis, R.; Sant, M.; Coleman, M.P.; Francisci, S.; Baili, P.; Pierannunzio, D.; Trama, A.; Visser, O.; Brenner, H.; Ardanaz, E.; et al. Cancer survival in Europe 1999-2007 by country and age: Results of EUROCARE-5-A population-based study. Lancet Oncol. 2014, 15, 23-34. [CrossRef]

5. Lianos, G.D.; Christodoulou, D.K.; Katsanos, K.H.; Katsios, C.; Glantzounis, G.K. Minimally Invasive Surgical Approaches for Pancreatic Adenocarcinoma: Recent Trends. J. Gastrointest. Cancer 2017, 48, 129-134. [CrossRef] [PubMed]

6. Griffin, J.F.; Poruk, K.E.; Wolfgang, C.L. Pancreatic cancer surgery: Past, present, and future. Chin. J. Cancer Res. 2015, 27, 332-348. [PubMed]

7. Halbrook, C.J.; Lyssiotis, C.A. Employing Metabolism to Improve the Diagnosis and Treatment of Pancreatic Cancer. Cancer Cell 2017, 31, 5-19. [CrossRef] [PubMed]

8. Frank, T.S.; Sun, X.; Zhang, Y.; Yang, J.; Fisher, W.E.; Gingras, M.C.; Li, M. Genomic profiling guides the choice of molecular targeted therapy of pancreatic cancer. Cancer Lett. 2015, 363, 1-6. [CrossRef] [PubMed]

9. Kiyotani, K.; Chan, H.T.; Nakamura, Y. Immunopharmacogenomics towards personalized cancer immunotherapy targeting neoantigens. Cancer Sci. 2018, 109, 542-549. [CrossRef] [PubMed]

10. Venkatakrishnan, A.J.; Deupi, X.; Lebon, G.; Tate, C.G.; Schertler, G.F.; Babu, M.M. Molecular signatures of G-protein-coupled receptors. Nature 2013, 494, 185-194. [CrossRef] [PubMed]

11. Zhou, H.; Rigoutsos, I. The emerging roles of GPRC5A in diseases. Oncoscience 2014, 1, 765-776. [CrossRef] [PubMed]

12. Jahny, E.; Yang, H.; Liu, B.; Jahnke, B.; Lademann, F.; Knosel, T.; Rummele, P.; Grutzmann, R.; Aust, D.E.; Pilarsky, C.; et al. The G Protein-Coupled Receptor RAI3 Is an Independent Prognostic Factor for Pancreatic Cancer Survival and Regulates Proliferation via STAT3 Phosphorylation. PLoS ONE 2017, 12, e0170390. [CrossRef] [PubMed]

13. Solinski, H.J.; Gudermann, T.; Breit, A. Pharmacology and signaling of MAS-related G protein-coupled receptors. Pharmacol. Rev. 2014, 66, 570-597. [CrossRef] [PubMed] 
14. Gentry, P.R.; Sexton, P.M.; Christopoulos, A. Novel Allosteric Modulators of G Protein-coupled Receptors. J. Biol. Chem. 2015, 290, 19478-19488. [CrossRef] [PubMed]

15. Scholz, N.; Gehring, J.; Guan, C.; Ljaschenko, D.; Fischer, R.; Lakshmanan, V.; Kittel, R.J.; Langenhan, T. The adhesion GPCR latrophilin/CIRL shapes mechanosensation. Cell Rep. 2015, 11, 866-874. [CrossRef] [PubMed]

16. Ferre, S.; Casado, V.; Devi, L.A.; Filizola, M.; Jockers, R.; Lohse, M.J.; Milligan, G.; Pin, J.P.; Guitart, X. $\mathrm{G}$ protein-coupled receptor oligomerization revisited: Functional and pharmacological perspectives. Pharmacol. Rev. 2014, 66, 413-434. [CrossRef] [PubMed]

17. Kumari, P.; Ghosh, E.; Shukla, A.K. Emerging Approaches to GPCR Ligand Screening for Drug Discovery. Trends Mol. Med. 2015, 21, 687-701. [CrossRef] [PubMed]

18. Kadara, H.; Fujimoto, J.; Men, T.; Ye, X.; Lotan, D.; Lee, J.S.; Lotan, R. A Gprc5a tumor suppressor loss of expression signature is conserved, prevalent, and associated with survival in human lung adenocarcinomas. Neoplasia 2010, 12, 499-505. [CrossRef] [PubMed]

19. Tao, Q.; Fujimoto, J.; Men, T.; Ye, X.; Deng, J.; Lacroix, L.; Clifford, J.L.; Mao, L.; Van Pelt, C.S.; Lee, J.J.; et al. Identification of the retinoic acid-inducible Gprc5a as a new lung tumor suppressor gene. J. Natl. Cancer Inst. 2007, 99, 1668-1682. [CrossRef] [PubMed]

20. Liu, S.; Ye, D.; Wang, T.; Guo, W.; Song, H.; Liao, Y.; Xu, D.; Zhu, H.; Zhang, Z.; Deng, J. Repression of GPRC5A is associated with activated STAT3, which contributes to tumor progression of head and neck squamous cell carcinoma. Cancer Cell Int. 2017, 17, 34. [CrossRef] [PubMed]

21. Chester, C.; Maecker, H.T. Algorithmic Tools for Mining High-Dimensional Cytometry Data. J. Immunol. 2015, 195, 773-779. [CrossRef] [PubMed]

22. Liu, B.; Yang, H.; Taher, L.; Denz, A.; Grutzmann, R.; Pilarsky, C.; Weber, G.F. Identification of Prognostic Biomarkers by Combined mRNA and miRNA Expression Microarray Analysis in Pancreatic Cancer. Transl. Oncol. 2018, 11, 700-714. [CrossRef] [PubMed]

23. Zhang, F.; Wen, Y.; Guo, X. CRISPR/Cas9 for genome editing: Progress, implications and challenges. Hum. Mol. Genet. 2014, 23, R40-R46. [CrossRef] [PubMed]

24. Binenbaum, Y.; Na'ara, S.; Gil, Z. Gemcitabine resistance in pancreatic ductal adenocarcinoma. Drug Resist. Updat. 2015, 23, 55-68. [CrossRef] [PubMed]

25. Garrido-Laguna, I.; Hidalgo, M. Pancreatic cancer: From state-of-the-art treatments to promising novel therapies. Nat. Rev. Clin. Oncol. 2015, 12, 319-334. [CrossRef] [PubMed]

26. Domoto, T.; Pyko, I.V.; Furuta, T.; Miyashita, K.; Uehara, M.; Shimasaki, T.; Nakada, M.; Minamoto, T. Glycogen synthase kinase-3beta is a pivotal mediator of cancer invasion and resistance to therapy. Cancer Sci. 2016, 107, 1363-1372. [CrossRef] [PubMed]

27. Roberts, R.A.; Nebert, D.W.; Hickman, J.A.; Richburg, J.H.; Goldsworthy, T.L. Perturbation of the mitosis/apoptosis balance: A fundamental mechanism in toxicology. Toxicol. Soc. 1997, 38, 107-115. [CrossRef]

28. Beurel, E.; Grieco, S.F.; Jope, R.S. Glycogen synthase kinase-3 (GSK3): Regulation, actions, and diseases. Pharmacol. Ther. 2015, 148, 114-131. [CrossRef] [PubMed]

29. Tejeda-Munoz, N.; Robles-Flores, M. Glycogen synthase kinase 3 in Wnt signaling pathway and cancer. IUBMB Life 2015, 67, 914-922. [CrossRef] [PubMed]

30. Ougolkov, A.V.; Fernandez-Zapico, M.E.; Savoy, D.N.; Urrutia, R.A.; Billadeau, D.D. Glycogen synthase kinase-3beta participates in nuclear factor kappaB-mediated gene transcription and cell survival in pancreatic cancer cells. Cancer Res. 2005, 65, 2076-2081. [CrossRef] [PubMed]

31. Wilson, W., 3rd; Baldwin, A.S. Maintenance of constitutive IkappaB kinase activity by glycogen synthase kinase-3alpha/beta in pancreatic cancer. Cancer Res. 2008, 68, 8156-8163. [CrossRef] [PubMed]

32. Shimasaki, T.; Ishigaki, Y.; Nakamura, Y.; Takata, T.; Nakaya, N.; Nakajima, H.; Sato, I.; Zhao, X.; Kitano, A.; Kawakami, K.; et al. Glycogen synthase kinase 3beta inhibition sensitizes pancreatic cancer cells to gemcitabine. J. Gastroenterol. 2012, 47, 321-333. [CrossRef] [PubMed]

33. Kitano, A.; Shimasaki, T.; Chikano, Y.; Nakada, M.; Hirose, M.; Higashi, T.; Ishigaki, Y.; Endo, Y.; Takino, T.; Sato, H.; et al. Aberrant glycogen synthase kinase 3beta is involved in pancreatic cancer cell invasion and resistance to therapy. PLoS ONE 2013, 8, e55289. [CrossRef] [PubMed] 
34. Pei, H.; Li, L.; Fridley, B.L.; Jenkins, G.D.; Kalari, K.R.; Lingle, W.; Petersen, G.; Lou, Z.; Wang, L. FKBP51 affects cancer cell response to chemotherapy by negatively regulating Akt. Cancer Cell 2009, 16, 259-266. [CrossRef] [PubMed]

35. Badea, L.; Herlea, V.; Dima, S.O.; Dumitrascu, T.; Popescu, I. Combined gene expression analysis of whole-tissue and microdissected pancreatic ductal adenocarcinoma identifies genes specifically overexpressed in tumor epithelia. Hepato Gastroenterol. 2008, 55, 2016-2027.

36. Zhang, G.; He, P.; Tan, H.; Budhu, A.; Gaedcke, J.; Ghadimi, B.M.; Ried, T.; Yfantis, H.G.; Lee, D.H.; Maitra, A.; et al. Integration of metabolomics and transcriptomics revealed a fatty acid network exerting growth inhibitory effects in human pancreatic cancer. Clin. Cancer Res. 2013, 19, 4983-4993. [CrossRef] [PubMed]

37. Barry, S.; Chelala, C.; Lines, K.; Sunamura, M.; Wang, A.; Marelli-Berg, F.M.; Brennan, C.; Lemoine, N.R.; Crnogorac-Jurcevic, T. S100P is a metastasis-associated gene that facilitates transendothelial migration of pancreatic cancer cells. Clin. Exp. Metastasis 2013, 30, 251-264. [CrossRef] [PubMed]

38. Wang, J.; Paris, P.L.; Chen, J.; Ngo, V.; Yao, H.; Frazier, M.L.; Killary, A.M.; Liu, C.G.; Liang, H.; Mathy, C.; et al. Next generation sequencing of pancreatic cyst fluid microRNAs from low grade-benign and high grade-invasive lesions. Cancer Lett. 2015, 356, 404-409. [CrossRef] [PubMed]

39. Donahue, T.R.; Tran, L.M.; Hill, R.; Li, Y.; Kovochich, A.; Calvopina, J.H.; Patel, S.G.; Wu, N.; Hindoyan, A.; Farrell, J.J.; et al. Integrative survival-based molecular profiling of human pancreatic cancer. Clin. Cancer Res. 2012, 18, 1352-1363. [CrossRef] [PubMed]

40. Frampton, A.E.; Castellano, L.; Colombo, T.; Giovannetti, E.; Krell, J.; Jacob, J.; Pellegrino, L.; Roca-Alonso, L.; Funel, N.; Gall, T.M.; et al. MicroRNAs cooperatively inhibit a network of tumor suppressor genes to promote pancreatic tumor growth and progression. Gastroenterology 2014, 146, 268-277. [CrossRef] [PubMed]

41. Thul, P.J.; Akesson, L.; Wiking, M.; Mahdessian, D.; Geladaki, A.; Ait Blal, H.; Alm, T.; Asplund, A.; Bjork, L.; Breckels, L.M.; et al. A subcellular map of the human proteome. Science 2017, 356, eaal3321. [CrossRef] [PubMed]

42. Chandrashekar, D.S.; Bashel, B.; Balasubramanya, S.A.H.; Creighton, C.J.; Ponce-Rodriguez, I.; Chakravarthi, B.; Varambally, S. UALCAN: A Portal for Facilitating Tumor Subgroup Gene Expression and Survival Analyses. Neoplasia 2017, 19, 649-658. [CrossRef] [PubMed]

43. Ran, F.A.; Hsu, P.D.; Wright, J.; Agarwala, V.; Scott, D.A.; Zhang, F. Genome engineering using the CRISPR-Cas9 system. Nat. Protoc. 2013, 8, 2281-2308. [CrossRef] [PubMed]

44. Sanjana, N.E.; Shalem, O.; Zhang, F. Improved vectors and genome-wide libraries for CRISPR screening. Nat. Methods 2014, 11, 783-784. [CrossRef] [PubMed]

(C) 2018 by the authors. Licensee MDPI, Basel, Switzerland. This article is an open access article distributed under the terms and conditions of the Creative Commons Attribution (CC BY) license (http:/ / creativecommons.org/licenses/by/4.0/). 